\title{
Cutaneous Squamous Cell Carcinoma with Mucinous Metaplasia in Dogs
}

\author{
Alex dos Santos, Marcella Barrella Ambrosio, Mariana Martins Flores \& Glaucia Denise Kommers
}

\begin{abstract}
Background: Squamous cell carcinoma (SCC) is one of the most common malignant skin tumors in domestic animals. Histologically, they are characterized by a proliferation of neoplastic keratinocytes with varied keratin production. Some SCCs have peculiar histological characteristics that permit them to be classified into uncommon to rare histological subtypes, reported in animals and humans. However, according to the authors' knowledge, the mucin-producing subtype described in humans has not yet been reported in animals. In this study, we report the occurrence of two mucin-producing SCCs in dogs, a histological presentation similar to that seen in cutaneous SCCs with mucinous metaplasia in humans.

Cases: Two dogs, a 5-year-old Yorkshire female and a 17-year-old Dachshund male, had a skin nodule near the tail and on the right eyelid. The nodules varied from 1 to $5 \mathrm{~cm}$ in diameter, were firm and covered with skin and hair. The cut surface was firm and white. Histological findings were compatible with squamous cell carcinoma, characterized by a neoplastic proliferation of keratinocytes originating in the epidermis and infiltrating the dermis. The keratinocytes were arranged in islands and occasional anastomosed cords, supported by a fibrous stroma. The formation of pearls varied from moderate to sparse. The nuclear and cellular pleomorphism was accentuated in case two and moderate in case one. Mitosis figures ranged from two to five in a high magnification field. Within the neoplasm, there were large vacuolated neoplastic cells with slightly fibrillar intracytoplasmic basophilic content. This content has been rarely observed in an extracellular medium. The presence of mucin was confirmed by positive Alcian Blue (AB) staining. In immunohistochemistry (IHC), tumor cells showed strong immunostaining for pancitokeratin, and in areas with marked mucin deposition, immunostaining was predominantly moderate to weak. No tumor cells were immunostained for CD34 and Bcl-2 antibodies. Compared to AB and Harris' hematoxylin, it was possible to demonstrate the presence of mucin in the cytoplasm of neoplastic keratinocytes using IHC. No vascular or lymphatic invasion by neoplastic cells was observed. The average cell proliferation index assessed by counting the nucleolar argyrophilic organizing regions (AgNOR) was 3.4 in case 1 and 4.5 in case 2 .

Discussion: Although the SCC routinely does not present a diagnostic challenge in veterinary practice, the histological presentation of the reported cases does not fit the current classification available in veterinary medicine. The histological presentation observed in these two dogs is similar to that described for cutaneous SCCs with mucinous metaplasia in humans, and so far not described in animals. The observation of intracytoplasmic mucin in humans is an essential finding for the diagnosis of SCC with mucin metaplasia. In the present cases, we observed a slightly basophilic amorphous substance in the cytoplasm of proliferated neoplastic keratinocytes, which stained strongly in blue when applied the Alcian Blue (AB) histochemical technique. This observation became more evident when using IHC counterstained with AB and Harris hematoxylin. In the histological analysis, the absence of an adenoid growth pattern or glandular formation amid neoplastic proliferation ruled out the possibility of a mucinous adenocarcinoma or a mucoepidermoid carcinoma. In addition, we could rule out a follicular neoplasia, including keratinizing infundibular acanthoma due to the absence of a central pore and the absence of immunostaining for CD34 and Bcl-2. These immunohistochemical findings, together with histological findings, reinforce the diagnosis of SCC with mucinous metaplasia in our dogs.
\end{abstract}

Keywords: canine, skin neoplasm, epidermoid cell carcinoma, mucin. 


\section{INTRODUCTION}

Squamous cell carcinoma (SCC) is one of the most common cutaneous malignancies in domestic animals. In addition, the SCCs consist of neoplastic keratinocytes associated with variable keratin production [9]. Some SCCs have peculiar histological characteristics that permit them to be classified as unusual to rare histological subtypes, reported in animals and humans [11]. However, according to the authors' knowledge, the mucin-producing subtype described in humans has not yet been reported in animals. The recognition of this histological type is important, since it has already been associated with a more aggressive biological behavior in humans [6]. Studies on the description and characterization of new histological variants of SCCs in animals are essential, as they may allow future correlations between certain histological subtypes and biological behavior.

In the present study, we report the occurrence of two mucin-producing SCCs in dogs, a histological presentation similar to that observed in cutaneous SCCs with mucinous metaplasia in humans $[2,6]$.

\section{CASES}

The necropsy and biopsy reports of the Laboratório de Patologia Veterinária of the Universidade Federal de Santa Maria (LPV-UFSM) were reviewed from 2000 to 2019. Over this period, 247 cases of cutaneous SCCs in dogs were diagnosed, of which 2 cases had the presence of intratumoral mucin. We retrieved the paraffin blocks from these 2 cases and a $3 \mu \mathrm{m}$-thick sections were cut and stained with hematoxylin (Harris hematoxylin) ${ }^{1}$ and $e^{2} \sin ^{2}$ for histologic reevaluation. It was performed Alcian Blue $\mathrm{pH} 2.5(\mathrm{AB})^{2}$ for mucin identification. Also we evaluated the cell proliferation by counting the nucleolar argyrophilic organizing regions $(\mathrm{AgNOR})^{2}$.

The immunohistochemistry (IHC) technique was applied in both cases. For antibodies CD34 (clone QBEnd/10), at dilution 1:200 and Bcl-24 (clone 124), at dilution 1:300, the technique was perfomed in a private laboratory. For pancitokeratin, the technique was perfomed in the Laboratório de Patologia Veterinária (LPV) -UFSM. IHC was performed with the polymer $\operatorname{method}^{5}$ (EasyLink One-HRP, EP-12-20502) using a rabbit polyclonal anti-bovine pan-cytokeratin antibo$\mathrm{dy}^{4}$, at a 1:2,000 dilution, modified from Masuda et al . [15]. Counterstaining was performed with $\mathrm{AB}$ (20 min) and Harris' hematoxylin. For $\mathrm{AgNOR}^{2}$, we stained the histological sections in solution containing a mixture of $2 \%$ gelatin and $1 \%$ formic acid in deionized water and $50 \%$ silver nitrate solution in a 1:2 ratio, respectively. The slides were immersed in this solution for 30 $\mathrm{min}$ at room temperature. Then, they were washed in deionized water, dehydrated, clarified, and mounted with synthetic medium. The black dots inside the nuclei were manually counted in 100 tumor cells, in random and representative fields by using oil immersion lens $(1000 \times)$. The frequency was obtained by dividing the number of AgNOR's by 100.

Case 1. A 5-year-old female Yorkshire terrier (received in 2011) with a 3-month history of a single cutaneous nodule close to the tail. The nodule was 1 $\mathrm{cm}$ in diameter, firm, covered with haired skin. It was white and well-demarcated on the cut surface.

Case 2. A 17-year-old male Dachshund (received in 2015) with a cutaneous lesion around the right lower eyelid with a year of evolution, which evolved to swelling and ulceration in the last 8 months. The 5.5 $\times 5.0 \times 3.5 \mathrm{~cm}$ nodule was partially covered by haired skin with a central irregular ulcer. The cut surface was firm, multilobulated, and white.

Histologically, the tumors were composed of a neoplastic proliferation of keratinocytes originating in the epidermis and invading the dermis (Figure 1). The keratinocytes were arranged in islands and occasional anastomosing cords, supported by a fibrous stroma. These cells were polyhedral, with abundant eosinophilic cytoplasm and indistinct cytoplasmic limits. The nuclei were round to oval, central, containing loose chromatin. The nucleoli were conspicuous. The formation of keratin pearls in the middle of proliferated keratinocytes varied from moderate to sparse. Nuclear and cellular pleomorphism was accentuated in case 2 and moderate in case 1 . The mitosis figures ranged from $2-5$ in a high power field $(400 \times)$. In case 2 , there were areas of moderate multifocal necrosis. Within the neoplasm, there were large vacuolated neoplastic cells with a slightly fibrillar intracytoplasmic basophilic content, sometimes distending the cytoplasm and displacing the nucleus to the periphery. This content has been rarely observed extracellularly, forming small pools of mucin in between the epithelial cells. However, these areas were closely associated with the proliferating neoplastic squamous component. The presence of intracytoplasmic and, rarely, extracellular 
mucin was confirmed by positive $\mathrm{AB}^{2}$ staining (Figures 2 and 3). In IHC, we observed a strong immunostaining for cytokeratin in epithelial squamous cells, while mucinous cells exhibited a more heterogeneous pattern of immunostaining. In areas with marked mucin deposition, immunostaining was predominantly moderate to weak. Using the IHC counterstained with $\mathrm{AB}^{2}$ and Harris' hematoxylin ${ }^{1}$, we could demonstrate the presence of mucin in the cytoplasm of neoplastic keratinocytes (Figure 4). For CD $34^{3}$ and Bcl-2 ${ }^{4}$ antibodies, no tumor cells were immunostained (Figure 4 inset). The internal controls (endothelial cells) were positive for CD34. No vascular or lymphatic invasion by neoplastic cells was observed. The average cell proliferation index assessed by $\mathrm{AgNOR}^{2}$ in these 2 cases was 4.3 NORs per nucleus. The count was 3.4 and 4.5 in cases 1 and 2, respectively.

\section{DISCUSSION}

The histological presentation of the SCCs reported in this study, especially with regard to the presence of intracytoplasmic mucin in neoplastic keratinocytes, has not been mentioned as a cutaneous SCC subtype in the current veterinary literature [9]. These changes are quite consistent with those observed in human cutaneous SCCs with mucinous metaplasia [2,6] and, to the authors' knowledge, there are no reports of this condition in dogs. The metaplasia in these cases occurs in the transformation of neoplastic keratinocytes, non-secreting cells, into mucin-producing cells [8].

The few studies describing this histological subtype in humans highlighted that intracytoplasmic mucin is an essential finding in SCCs with mucinous metaplasia [2,6]. In the 2 dogs included in the present report, it was observed an amorphous substance in the cytoplasm of some neoplastic keratinocytes with strongly blue in $\mathrm{AB}$-stained sections. The $\mathrm{AB}$ staining is used in mucinous SCCs to prove that the content deposited in the cytoplasm of the neoplastic keratinocytes is mucin $[2,6]$.

Mucins are high molecular weight glycoproteins, related to cell signaling and immune response, expressed by epithelial cells [3]. In humans, mucin genes are classified into 2 types; secreted or membrane bound [16]. In the 2 dogs in the present study, the mucin observed in the keratinocytes was probably the membrane bound mucin, as only this type was associated with the development of SCCs in humans [10,13,20].
It is important to note that in the 2 dogs of the present study, mucin was present within neoplastic keratinocytes, cells that are not normally responsible for mucus production $[9,11]$. In addition, the distribution of mucin varied, being observed heterogeneously in the cytoplasm of multiple keratinocytes. Studies in humans have shown that approximately $30 \%$ of neoplastic keratinocytes have intracytoplasmic mucin, often with signet ring cells [2]. These cells, however, were not observed in the 2 dogs reported in the present study. In rare areas of the tumors, mucin was present extracellularly, possibly due to the rupture of keratinocytes. Moreover, there was a positive immunostaining for pancitokeratin, both in keratinocytes with intracytoplasmic mucin and in those without it. However, the intensity of the immunostaining was weaker in the mucin-containing keratinocytes. There are reports in humans that under the influence of environmental factors, the presence of bipotent parents can generate both differentiated keratinocytes and mucin-producing cells [18]. Therefore, the transformation of neoplastic keratinocytes into mucin-producing cells will likely cause these cells to gradually lose cytokeratin expression. This may suggest that the greater the deposition of intracytoplasmic mucin, the lower the immunostaining for cytokeratin, as observed in the present study.

Mucinous adenocarcinoma and mucoepidermoid carcinoma stand out among the main differential diagnoses for mucinous SCC in humans [2]. Mucinous adenocarcinoma is characterized histologically by an invasive SCC combined with an adenocarcinoma component consisting of glandular structures and cystic spaces lined by a mucin-secreting epithelium [2,21]. Conversely, mucoepidermoid carcinoma is an adnexal differentiation tumor, composed of polygonal squamous cells arranged in lobes, without an intraepidermal component. In the center of the lobes, there are many vacuolated and mucin-secreting cells [19]. In veterinary medicine, there has been a description of cutaneous mucoepidermoid carcinoma in a dog, with a few mucus-producing cells [14]. The absence of an adenoid growing pattern or glandular formation is an important feature in mucin-producing SCCs [2,6], and aided the diagnosis of SCC in the tumors described in this report.

Keratinizing infundibular acanthoma (IKA), a follicular neoplasia that affects dogs and that can present a stroma with a moderate amount of mucin, should be considered [11]. However, in the 2 cases described 


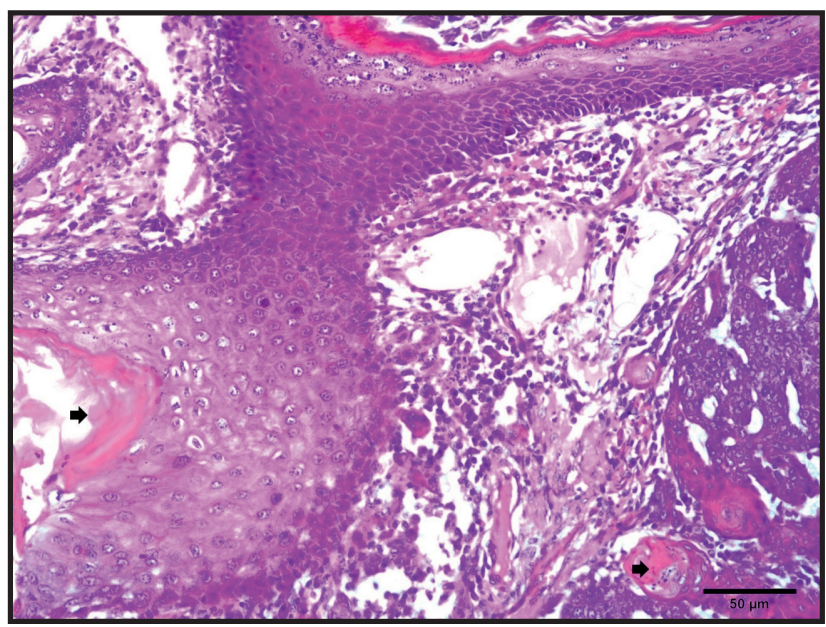

Figure 1. Skin. Neoplastic keratinocytes are observed originating in the epidermis, invading and infiltrating the dermis, with a laminated keratin production area (arrows). [HE 40x].

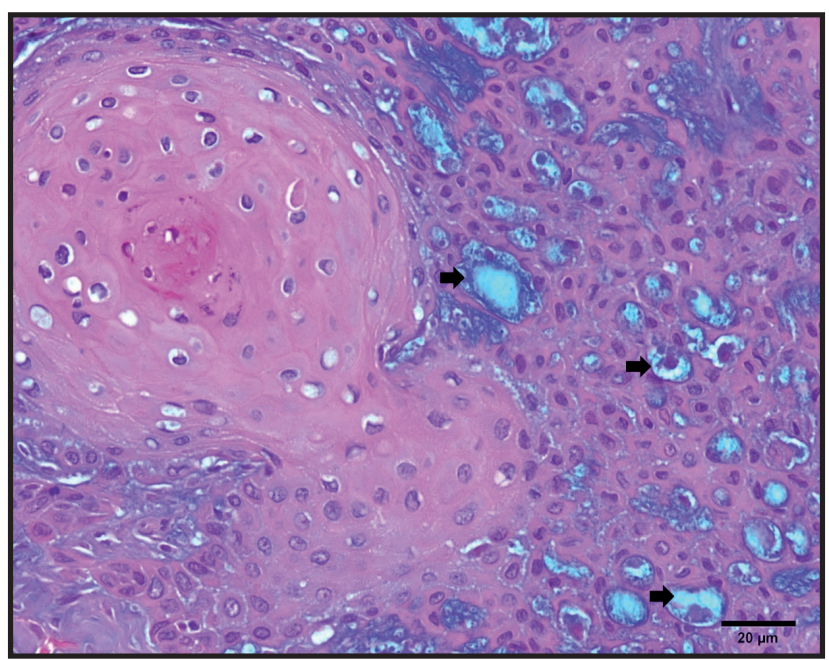

Figure 3. Skin. Neoplastic keratinocytes close to a keratin pearl with intracytoplasmic basophilic fibrillar mucinous content strongly stained in blue. The nuclei are condensed and sometimes moved to the periphery (arrows). [Alcian Blue 10x; bar= $20 \mu \mathrm{m}$ ].

here, mucin was present in the cytoplasm of neoplastic keratinocytes, which was better evidenced in IHC counterstained with $\mathrm{AB}$ and hematoxylin. This phenotypic and histochemical finding excludes an IKA, in which mucin is produced by dermal fibroblasts present in the tumor stroma [11]. In addition, the absence of a central pore, which gives the IKA a feeling of a cup or volcano, and the nuclear and cellular pleomorphism observed in our 2 cases also rule out this possibility [9]. In addition, the AgNOR count in the 2 studied SCC cases is within the standards described for the cell proliferation index in SCCs in humans [1] and cattle [15]; it is within the average described for malignant skin tumors (varies from 3.38 to 6.98) [17] and it is higher than the AgNOR count in IKAs diagnosed in the Laboratório de Patologia Veterinária (LPV) - UFSM (unpublished data).

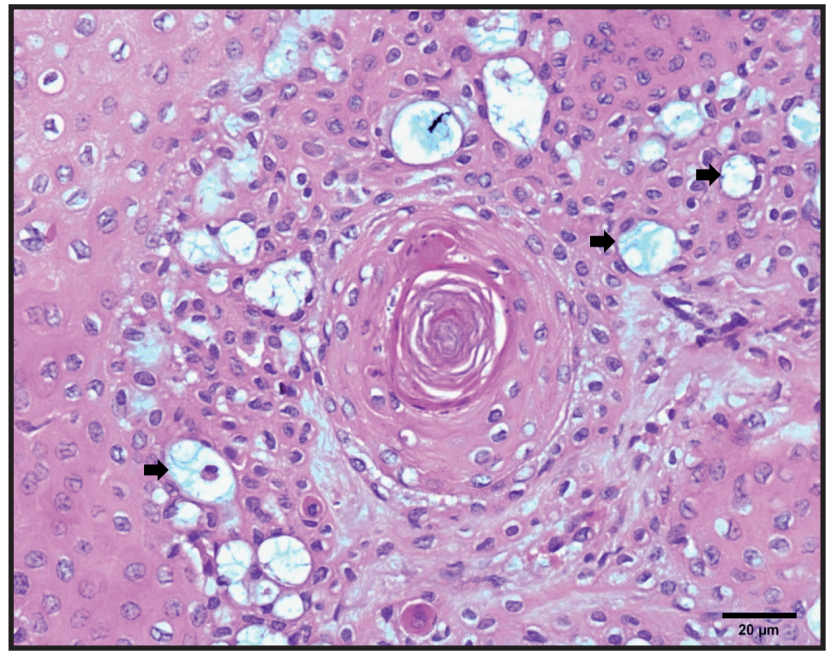

Figure 2. Skin. Neoplastic keratinocytes are observed with vacuolated cytoplasm and deposition of basophilic fibrillar mucinous content, sometimes displacing the nucleus toward the periphery (arrows). In the center is a pearl of keratin. [HE $40 \times$; bar $=50 \mu \mathrm{m}$ ].



Figure 4. Skin. Neoplastic keratinocytes strongly immunostained for antipancitokeratin with intracytoplasmic mucinous content stained strongly in blue by Alcian Blue (arrows). [Polymer-HRP method 10x]. In set, antiCD34 negative tumor cells.

The IHC technique was essential in this work to exclude differential diagnoses. The use of pancitokeratin counterstained with $A B$ facilitated the observation of mucin in neoplastic keratinocytes. The absence of immunostaining for CD34 ruled out a follicular tumor [5]. This antibody is expressed in several follicular tumors, not being expressed in normal skin or in epidermal, sebaceous, or apocrine carcinomas [12]. Bcl-2 is an anti-apoptotic protein that plays an important role in the maintenance of stem cells. Its expression is increased in follicular tumors, mainly tricoblastoma [4] and is not expressed in the SCC in humans [7]. These immunohistochemical findings, together with the histological findings, reinforce the diagnosis of SCC and rule out any follicular neoplasia in the present 2 described cases. 
In conclusion, the similarity of the cases described here with those reported in humans and the absence of the SCC subtype with mucinous metaplasia in the classification available in veterinary medicine highlight the importance of this report. In humans, this SCC subtype tends to be more aggressive [11,21]. However, it would not be possible to infer prognostic factors here, considering the case series limited to 2 cases, which were not followed-up prospectively.

\section{MANUFACTURERS}

${ }^{1}$ Merck KGaA. Darmstadt, Germany.

${ }^{2}$ Laboratório de Patologia Veterinária, CCS, UFSM. Santa Maria,
RS, Brazil.

${ }^{3}$ Cell Marque Corporation. Rocklin, CA, USA.

${ }^{4}$ Dako - Agilent Technologies Inc. Carpinteria, CA, USA.

${ }^{5}$ Erviegas Química Fina e Plásticos Eireli. Indaiatuba, SP, Brazil.

Acknowledgments. This research was financially supported by Coordenação de Aperfeiçoamento de Pessoal de Nível Superior (CAPES), Brazil; A. Santos has a Doctor's scholarship from CAPES. This research was also financially supported by Fundação de Amparo à Pesquisa do Estado do Rio Grande do Sul (FAPERGS; Grant \#19/2551-0001817-7).

Declaration of interest. The authors declare no conflicts of interest. The authors alone are responsible for the content and writing of this paper.

\section{REFERENCES}

1 Aroni K., Mastoraki A., Kyriazi E., Ioannidis E. \& Patsouris E. 2007. Silver-stained organizer regions and immunoglobulins in cutaneous keratoacanthomas and squamous cell carcinomas. Pathology Research and Practice. 203(9): 659-665.

2 Caputo V.M.D., Colombi R.M.D., Ribotta M.M.D. \& Rongioletti F.M.D. 2011. Cutaneous squamous cell carcinoma with mucinous metaplasia on the sole associated with high-risk human papillomavirus type 18. The American Journal of Dermatopathology. 33(3): 317-322.

3 Chakraborty S., Bonthu N., Swanson B.J. \& Batra S.K. 2011. Role of Mucins in the Skin during Benign and Malignant Conditions. Cancer Letters. 301(2): 127-141.

4 Córdoba A., Guerrero D., Larrinaga B., Iglesias M.E., Arrechea M.A. \& Yanguas J.I. 2009. Bcl-2 and CD10 expression in the differential diagnosis of trichoblastoma, basal cell carcinoma, and basal cell carcinoma with follicular differentiation. International Journal of Dermatology. 48(7): 713-717.

5 Firmo B.F., Grandil F., Cogliati B. \& Rocha N.S. 2014. CD34 Immunoexpression in canine skin follicular tumors and basal cell carcinomas: case series report. Source Journal of Veterinary Science. 1(1): 1-5.

6 Friedman K.J., Hood A.F. \& Farmer E.R. 1988. Cutaneous squamous cell carcinoma with mucinous metaplasia. Journal of Cutaneous Pathology. 15(3): 176-182.

7 Gaballah M. \& Alhmed R.A. 2015. Diagnostic value of CD10 and Bcl2 expression in distinguishing cutaneous basal cell carcinoma from squamous cell carcinoma and seborrheic keratosis. Pathology - Research and Practice. 211(12): 931-938.

8 Giroux V. \& Rustgi A.K. 2017. Metaplasia: tissue injury adaptation and a precursor to the dysplasia-cancer sequence. Nature Reviews Cancer. 17(10): 594-604.

9 Goldschmidt M.H. \& Goldschmidt K.H. 2017. Epithelial and Melanocytic Tumors of the Skin. In: Meuten D.J. (Ed). Tumors of Domestic Animals. 5th edn. Ames: John Wiley \& Sons, pp.89-102.

10 Groce M.V., Rabassa M.E., Price M.R. \& Segal-Eiras A.2001. MUC1 Mucin and Carbohydrate Associated Antigens as Tumor Markers in Head and Neck Squamous Cell Carcinoma. Pathology Oncology Research. 7(4): 284-291.

11 Gross T.L., Ihrke P.J., Walder E.J. \& Affolter V.K. 2005. Skin Diseases of the Dog and Cat: Clinical and Histopathologic Diagnosis. 2nd edn. Ames: Blackwell Science Ltd., pp.581-588.

12 Kok M.K., Chambers J.K., Ong S.M., Nakayama H. \& Uchida K. 2018. Hierarchical cluster analysis of cytokeratins and stem cell expression profiles of canine cutaneous epithelial tumors. Veterinary Pathology. 55(6): 821-837.

13 Mahomed F. 2011. Recent advances in mucin immunohistochemistry in salivary gland tumors and head and neck squamous cell carcinoma. Oral Oncology. 47(9): 797-803. 
14 Martínez C.M., Buendía A.J., Sánchez J., Vilafranca M., Altimira J., Ramirez G., Garcia B. \& Navarro J.A. 2009. Histopathological and immunophenotypical characterization of a combined melanoma and mucoepidermoid carcinoma in a dog. Anales de Veterinaria de Murcia. 25: 135-142.

15 Masuda E.K., Kommers G.D., Martins T.B., Barros C.S.L. \& Piazer J.V.M. 2011. Morphological Factors as Indicators of Malignancy of Squamous Cell Carcinomas in Cattle Exposed Naturally to Bracken Fern (Pteridium aquilinum). Journal Comparative Pathology. 144(1): 48-54.

16 Moniaux N., Escande F., Porchet N., Aubert J.P. \& Batra S.K. 2001. Structural organization and classification of the human mucin genes. Frontiers in Bioscience. 6: 1192-1206.

17 Palanivelu M., Lakkawar A.W., Varshney K.C., Kumar R. \& Kumar M.A. 2013. Histochemical Assessment of AgNORs in Cutaneous Neoplasms of Dogs. Advances in Animal and Veterinary Sciences. 1(3): 93-95.

18 Papini S., Cecchetti D., Capani D., Fitzgerald W., Grivel J.C., Chen S., Margolis L. \& Revoltella R.P. 2003. Isolation a clonal analysis of human epidermal keratinocyte stem cells in long-term culture. Stem Cells. 21(4): 481-494.

19 Riedlinger W.F.J., Hurley Y., Dehneer L.P. \& Lind A.C. 2005. Mucoepidermoid carcinoma of the skin: a distinct entity from adenosquamous carcinoma. The American Journal of Surgical Pathology. 29(1): 131-135.

20 Thakur A., Tupkari J.V., Joy T., Kende P.P., Siwach P. \& Ahire M.S. 2018. Expression of mucin-1 in oral squamous cell carcinoma and normal oral mucosa: An immunohistochemical study. Journal of Oral and Maxillofacial Pathology. 22(2): 210-215.

21 Weidner N. \& Foucar E. 1985. Adenosquamous carcinoma of the skin and aggressive mucin and gland-forming squamous carcinoma. Archives of Dermatology. 121(6): 775-779. 